\title{
Regulating bacterial gene expression with small molecules by altering DNA supercoiling
}

\author{
Soumya G. Remesh ${ }^{1}$, Subhash Verma², Zhong Qian², Sankar Adhya², Michal Hammel ${ }^{1}$ \\ ${ }^{1}$ Molecular Biophysics and Integrated Biology, Lawrence Berkeley National Laboratory, Berkeley, CA - 94720 \\ ${ }^{2}$ National Cancer Institute, National Institutes of Health, 37 Convent Drive, BLDG 37 RM 5138 \\ Bethesda MD USA \\ sgremesh@lbl.gov.
}

Bacterial chromosome (nucleoid) organization is dependent on conserved nucleoid associated proteins (NAPs), mainly the histone-like protein $\mathrm{HU}$ involving interactions spanning the nanoscale and mesoscale to direct DNA supercoiling. By means of macromolecular crystallography, small angle X-ray scattering (SAXS) and soft X-ray tomography (SXT) we showed that DNA dependent HU networking controls DNA supercoiling to maintain the nucleoid structure. Moreover, we characterized the molecular switch of gain-of-function HU mutations that altered DNA supercoiling and reprograms Escherichia coli to an invasive form. The fact that DNA supercoiling also controls expression of virulence factors in many pathogens makes $\mathrm{HU}$ an attractive target for novel antibacterial agents. We showed that the HU networking can be disrupted by small molecules and revealed new molecular mechanism to regulate gene expression. Using a virtual screening of a small molecule fragment library, we identified a bioactive molecule that targets the HU-HU networking interface. Our structural characterization of the molecular mechanism of this prototypic small molecule has validated the $\mathrm{HU}$ networking as a bona-fide target for regulating bacterial gene expression. Using our integrated structural biology

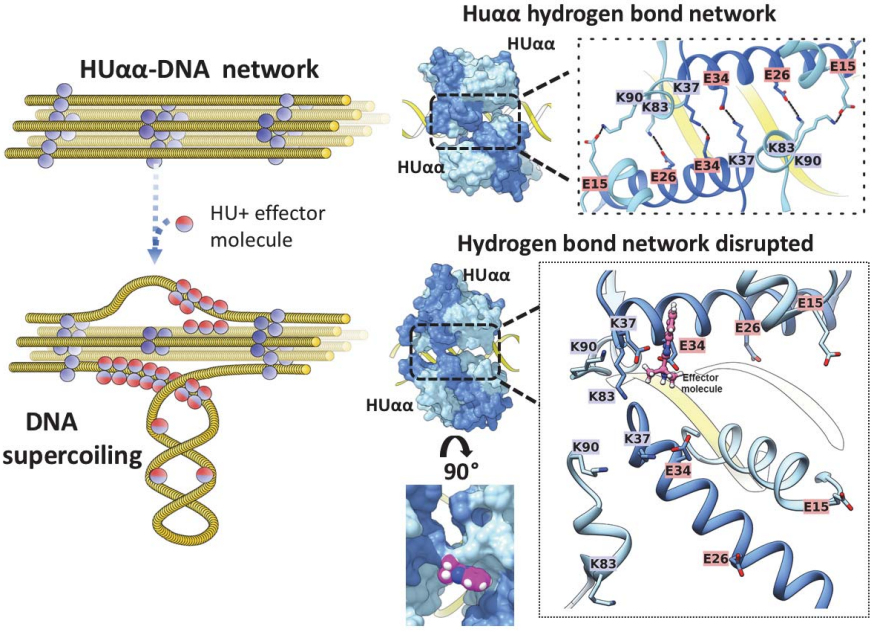

HU multimerization and HU-DNA network directs bacterial DNA supercoiling. We show that addition of a prototypic effector molecule disrupts critical hydrogen bond network altering DNA supercoiling. approach the mechanistic insights we provide underpins a rational strategy for the discovery of new antimicrobial agents that re-program gene expression in the pathogenic state.

1. HU multimerization shift controls nucleoid compaction. Science Adv. 2016;2(7):e1600650

2. Conversion of commensal Escherichia coli K- 12 to an invasive form via expression of a mutant histone-like protein. MBio. 2011;2(5)

3. Nucleoid remodeling by an altered HU protein: reorganization of the transcription program. Proc Natl Acad Sci U S A. 2005;102(45):16397-402

4. DNA supercoiling is a fundamental regulatory principle in the control of bacterial gene expression. Biophys Rev. 2016;8(3):209-20

5. Characterization of porin and ompR mutants of a virulent strain of Salmonella typhimurium: ompR mutants are attenuated in vivo. Infect Immun. 1989;57(7):2136-40 